\title{
When do we simulate non-human agents? Dissociating communicative and non-communicative actions
}

\author{
Roman Liepelt ${ }^{\mathrm{a}, *}$, Wolfgang Prinz ${ }^{\mathrm{a}}$, Marcel Brass ${ }^{\mathrm{b}}$ \\ ${ }^{a}$ Max Planck Institute for Human Cognitive and Brain Sciences, Department of Psychology, Leipzig, Germany \\ ${ }^{\mathrm{b}}$ Ghent University, Department of Experimental Psychology and Ghent Institute for Functional and Metabolic Imaging, Ghent, Belgium
}

\section{A R T I C L E I N F O}

\section{Article history:}

Received 10 August 2009

Revised 29 January 2010

Accepted 1 March 2010

\section{Keywords:}

Simulation

Motor priming

Communicative action

Non-human agent

\begin{abstract}
A B S T R A C T
There is strong evidence that we automatically simulate observed behavior in our motor system. Previous research suggests that this simulation process depends on whether we observe a human or a non-human agent. Measuring a motor priming effect, this study investigated the question of whether agent-sensitivity of motor simulation depends on the specific action observed. Participants saw pictures depicting end positions of different actions on a screen. All postures featured either a human or non-human agent. Participants had to produce the matching action with their left or right hand depending on the hand presented on the screen. Three different actions were displayed: a communicative action (emblem), a transitive (goal-directed) action and an intransitive action. We found motor priming effects of similar size for human and non-human agents for transitive and intransitive actions. However, the motor priming effect for communicative actions was present for the human agent, but absent for the non-human agent. These findings suggest that biological tuning of motor simulation is highly action-selective and depends on whether the observed behavior appears to be driven by a reasonable goal.
\end{abstract}

(c) 2010 Elsevier B.V. All rights reserved.

\section{Introduction}

An extensive body of brain imaging research suggests that passive observation of human action leads to activation of brain areas that are also involved in motor preparation and execution (Buccino et al., 2001; Fogassi et al., 2005; Gallese, Fadiga, Fogassi, \& Rizzolatti, 1996; Gazzola, Aziz-Zadeh, \& Keysers, 2006; Grezes, Armony, Rowe, \& Passingham, 2003; Iacoboni et al., 1999). Furthermore, behavioral research strongly suggests that the observation of an action activates a corresponding motor representation in the observer. This phenomenon has been termed motor simulation (for an overview see Brass and Heyes, 2005 and Blakemore and Frith, 2005). Motor simulation

\footnotetext{
* Corresponding author. Address: Max Planck Institute for Human Cognitive and Brain Sciences, Department of Psychology, Stephanstr. 1A, 04103 Leipzig, Germany. Tel.: +49 3419940 2279; fax: +49 3419940 2204.

E-mail address: liepelt@cbs.mpg.de (R. Liepelt).
}

is typically measured with a motor priming paradigm, in which participants have to carry out an instructed response while observing task-irrelevant congruent or incongruent actions (e.g., Brass, Bekkering, Wohlschläger, \& Prinz, 2000). It has been demonstrated that the observation of a finger movement that corresponds to the instructed finger movement leads to facilitation of the response and observing a non-corresponding finger movement leads to interference (Bertenthal, Longo, \& Kosobud, 2006; Brass et al., 2000; Stürmer, Aschersleben, \& Prinz, 2003).

\section{Motor simulation of biological and non-biological agents}

One issue that is still controversial is whether motor simulation is restricted to observing human agents or also occurs for non-human agents (Gazzola, Rizzolatti, Wicker, \& Keysers, 2007; Kilner, Paulignan, \& Blakemore, 2003; Press, Bird, Flach, \& Heyes, 2005). A range of studies provide evi- 
dence that only actions produced by human agents lead to motor priming effects and not when these actions are produced by non-human agents (Kilner et al., 2003; Tai, Scherfler, Brooks, Sawamoto, \& Castiello, 2004; see also Tsai \& Brass, 2007). Other studies support the idea of attenuated motor priming for non-human movements (Liepelt \& Brass, 2010; Longo \& Bertenthal, 2009; Press, Gillmeister, \& Heyes, 2006; Press et al., 2005). There is, however, also neuroimaging evidence showing similar activation in the mirror system for human and non-human movement stimuli (Gazzola et al., 2007; see also Jansson, Wilson, Williams, \& Mon-Williams, 2007; Stanley, Gowen, \& Miall, 2007).

While the role of the observed agent has been extensively investigated, it is still an open question whether motor priming might also depend on the interaction of the agent and the specific movement executed by the agent. This, however, seems important, because not only humans, but also non-human agents might have a restricted range of actions that we perceive as a reasonable part of their action repertoire. Interestingly, there is one brain imaging study that has investigated whether we motorically simulate actions carried out by animals. This study revealed strong activation in motor-related areas whenever the observed behavior was also in the behavioral repertoire of the observer (e.g., biting) but not when it was not in his repertoire (Buccino et al., 2004). Furthermore, it has been demonstrated that the observation of biologically impossible movements does not lead to motor priming (Longo, Kosobud, \& Bertenthal, 2008; but see Candidi, Urgesi, Ionta, \& Aglioti, 2008). These studies seem to suggest that motor priming only occurs when the observed behavior is also in the behavioral repertoire of the observer. However, Liepelt and colleagues recently demonstrated that motor priming effects also depend on the interpretation of an observed behavior as intentional or unintentional (Liepelt, von Cramon, \& Brass, 2008). In this study, the observed behavior was in the behavioral repertoire of the observer but was not interpreted as intentionally carried out by the model. One potential way to further test this idea is to present a behavior that the observer would not consider as a reasonable part of the behavioral repertoire of the observed agent but is part of the behavioral repertoire of the observer. A robot, for example, might be able to produce a simple hand-closing gesture when grasping an object. However, one would not expect a robot to carry out a communicative gesture, such as a handshake, because you would not consider it as a reasonable behavior for a robot. This would suggest that motor simulation does not involve the simulation of the direct physical movement, but also of the goals of actions (Bekkering, Wohlschlager, \& Gattis, 2000; Ochiai, Mushiake, \& Tanji, 2005).

\section{The present study}

The aim of the present study was to test whether motor simulation is not only agent sensitive (human or non-human), but also sensitive to the specific action type performed by the agent. The experimental design was slightly different from the paradigms used in previous motor priming studies (e.g., Brass et al., 2000) and resembles to some extent a study on object affordances by Tucker and Ellis (1998). We developed movement stimuli for three different types of actions: a communicative action (emblem) showing a typical handshake gesture, a transitive (goal-directed) action showing a hand grasping an apple and an intransitive action showing a hand-closing gesture pursuing no further goal. These actions were either executed by a non-human agent or by a human agent. We predict that the motor priming effect should not differ for observing human or non-human agents as long as the observed behavior could be considered as a reasonable goal of the agent (i.e., simple transitive or intransitive movements). However, when the observed behavior cannot be considered a reasonable goal for the non-human agent (i.e., a communicative gesture) the motor priming effect should be largely reduced.

\section{Experiment 1}

\subsection{Method}

\subsubsection{Participants}

A group of 27 undergraduate students (14 males, age range: $21-28$, mean age: 23.8 , SD: 2.0 ) participated in this experiment. All participants were right-handed, had normal or corrected-to-normal vision, and were naive with regard to the hypotheses of the experiment. Participants were paid $€ 7$ for participation.

\subsubsection{Apparatus and stimuli}

Stimuli were presented on a 17-in. color monitor that was connected to a Pentium I PC. Experiments were carried out using ERTS software (Experimental Runtime System; Beringer, 2000). The stimuli consisted of one still picture of a right or left hand photographed from a third person perspective presented in the middle of the screen (see Fig. 1). The picture showed the end position of one of three gestures (communicative, intransitive, transitive). For the communicative gesture, the hand was shown in a typical handshake gesture. For the intransitive gesture, the final part of a hand closing movement was shown and for the transitive gesture, we showed a hand grasping an apple. The same gesture was presented depicting two different agents (human, wooden). After a time interval of $800 \mathrm{~ms}$, an additional number ( 1 or 2 ) was presented above the hand. The number always appeared in the same position. At a viewing distance of $80 \mathrm{~cm}$, the hand on the screen subtended a visual angle of $5.71^{\circ} \times 12.33^{\circ}$ (communicative), $4.22^{\circ} \times 7.97^{\circ}$ (intransitive), $7.27^{\circ} \times 8.39^{\circ}$ (transitive) and the number, $0.29^{\circ} \times 0.72^{\circ}$.

\subsubsection{Procedure and design}

An adapted version of the paradigm developed by Brass et al. (2000) was used in the present experiment. Participants had to release either the index finger of their left or right hand from a response board as indicated by a number (1: left index finger, 2: right index finger) and form one of three possible gestures (communicative, intransitive or transitive) corresponding to the gesture presented on the screen. Each gesture type was given in separate blocks. 


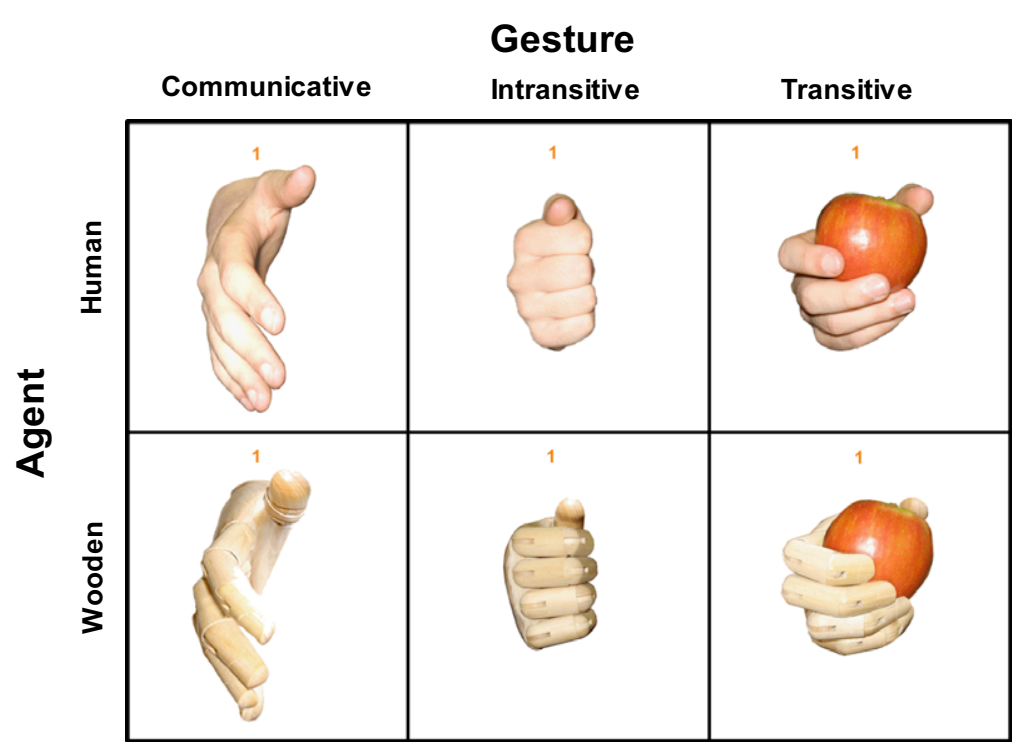

Fig. 1. The figures show the stimuli and design of two agents (human, wooden) and three gesture types (communicative, intransitive, transitive) used in Experiment 1 . All stimuli are shown from a third person perspective displaying a right hand in a mirror-congruent condition.

Depending on the presented hand stimulus (left or right hand), the response hand was lifted either congruently or incongruently in response to the number. In congruent trials, the observed hand and the required response hand were presented in a mirror perspective (e.g., left-hand response to the example hand cues shown in Fig. 1). In incongruent trials, the observed hand and the required response hand were presented in a complementary perspective (e.g., right-hand response to the example hand cues shown in Fig. 1). Participants were instructed to respond to the numbers irrespective of the observed hand (left or right hand) on the screen.

Each trial began with a frame lasting for 800 ms showing the hand stimulus in an end position. In the second frame, the same hand was presented and additionally a digit appeared above the stimulus hand for $1915 \mathrm{~ms}$. A tone that informed participants whether a response was measured (due to the correct use of the response board) was given for $50 \mathrm{~ms}$ followed by a blank screen for $2100 \mathrm{~ms}$. Each trial lasted $4865 \mathrm{~ms}$ in total (see Fig. 2).

Before running the experiment, we presented either a static real human hand or a static wooden analogue model of a hand to all participants. They were told that they would see pictures of the respective hand model in differ- ent gestures on the screen during the experiment. This statement was included in the general instructions without any special emphasis. Each gesture (communicative, intransitive, transitive) was presented for two blocks, each consisting of 64 trials, separated by a short break. Wooden and human hands were randomly presented in each of the blocks. The order of gesture types was counterbalanced across participants. Participant performed 384 trials in total.

\subsection{Results}

In all experiments prior to statistical analyses, all trials in which responses were incorrect $(1.4 \%)$ or outliers $(1.2 \%)$ that were three standard deviations above or below the mean group Reaction Times (RTs) were excluded from statistical RT analyses. Congruency effects for all conditions are presented in Fig. 3. For an overview of mean RTs and mean percent errors of all conditions of Experiment 1, including main effects, see Table 1.

\subsection{1. $R T$ analysis}

We used a $2 \times 3 \times 2$-factorial design including the 2-level factor Agent (human, wooden), the 3-level factor Ges-

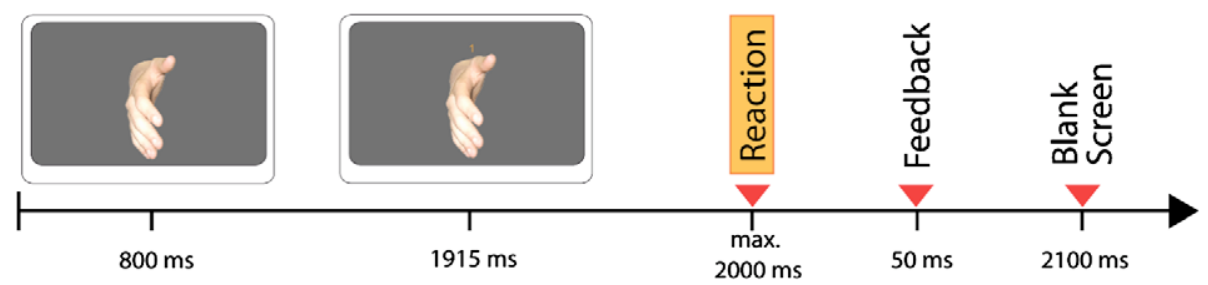

Fig. 2. Shown is the stimulus sequence for each trial as used in the present experiments. Each trial started with a picture (displayed for 800 ms) showing a static hand. In the second frame (displayed for $1915 \mathrm{~ms}$ ), the symbolic imperative stimulus ' 1 ' or ' 2 ' appeared centrally above the hand. Participants had to respond within $2000 \mathrm{~ms}$. The reaction was followed by a tone for $50 \mathrm{~ms}$ and a constant blank screen for $2100 \mathrm{~ms}$. 


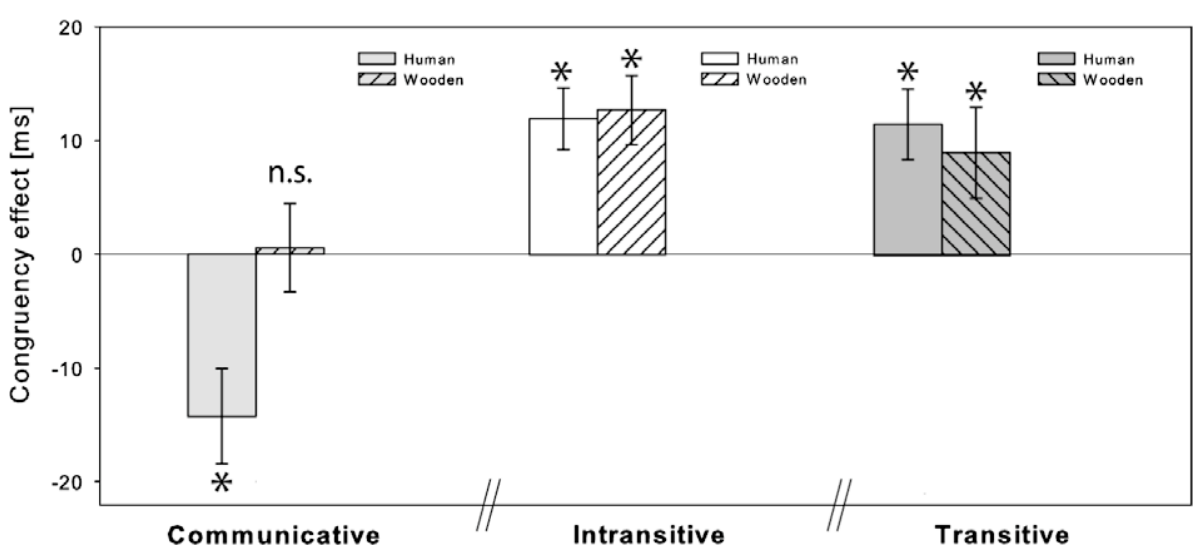

Fig. 3. Congruency effect (incongruent minus congruent) in milliseconds (ms) plotted with the SE of the congruency effect for Experiment 1 shown for communicative (left panel, light gray), intransitive (middle panel, white) and transitive gestures (right panel, dark gray) of the human agent (blank bars) and the wooden agent (striped bars).

Table 1

Mean reaction times (RTs) in milliseconds (ms) and percent errors (upper part) are shown for Agent (human, wooden), Gesture (communicative, intransitive, transitive) and Congruency (congruent, incongruent) conditions of Experiment 1. Corresponding $p$-values for within-subjects effects (lower part) are shown separately for RTs and percent errors.

\begin{tabular}{lll}
\hline Condition & RT (ms) & Error (\%) \\
\hline Human communicative congruent & 414 & 1.5 \\
Human communicative incongruent & 399 & 0.9 \\
Wooden communicative congruent & 405 & 1.3 \\
Wooden communicative incongruent & 405 & 0.7 \\
Human intransitive congruent & 375 & 1.4 \\
Human intransitive incongruent & 387 & 2.9 \\
Wooden intransitive congruent & 377 & 1.0 \\
Wooden intransitive incongruent & 390 & 2.2 \\
Human transitive congruent & 390 & 1.0 \\
Human transitive incongruent & 402 & 1.3 \\
Wooden transitive congruent & 394 & 0.7 \\
Wooden transitive incongruent & 403 & 1.3 \\
Within-subjects & $p(R T)$ & $p($ Error $)$ \\
\hline Agent $(\mathrm{A})$ & $n . s$. & $n . s$. \\
Gesture (G) & $<0.001^{\mathrm{b}}$ & $<0.05^{\mathrm{a}}$ \\
Congruency (C) & $<0.05^{\mathrm{a}}$ & $n . s$. \\
$\mathrm{A} \times \mathrm{G}$ & $n . s$. & $n . s$. \\
$\mathrm{A} \times \mathrm{C}$ & $n . s$. & $n . s$. \\
$\mathrm{G} \times \mathrm{C}$ & $<0.001^{\mathrm{b}}$ & $<0.05^{\mathrm{a}}$ \\
$\mathrm{A} \times \mathrm{G} \times \mathrm{C}$ & $<0.05^{\mathrm{a}}$ & $n . s$. \\
\hline $\mathrm{a}$ & &
\end{tabular}

a $\operatorname{Sign}(p<0.05)$

b $\operatorname{Sign}(p<0.001)$, n.s. $(p>0.05)$.

ture (communicative, intransitive, transitive), as well as the 2-level factor Congruency (congruent, incongruent), with all factors as within-subject variables (see Figs. 1 and 3). The same analysis was conducted for accuracy data.

In the present experiment, we observed no main effect of Agent, $F(1,26)=1.85, \mathrm{MSe}=83.16, p>0.05$, partial $\eta^{2}=0.07$. We found a main effect of Gesture, $F(2,52)=$ 11.57, $\mathrm{MSe}=1496.79, p<0.001$, partial $\eta^{2}=0.31$, with the fastest RTs for the intransitive gesture (383 ms), followed by the transitive gesture $(397 \mathrm{~ms})$ and slowest RTs for the communicative gesture (406 ms). Further, we found a significant effect of Congruency, $F(1,26)=7.86, \quad \mathrm{MSe}=$ $286.08, p<0.05$, partial $\eta^{2}=0.23$, due to faster RTs in congruent (392 ms) as compared to incongruent conditions
(398 ms). We found no significant effects of Agent $\times$ Gesture, $F(2,52)=1.37, \quad \mathrm{MSe}=106.90, \quad p>0.05$, partial $\eta^{2}=0.05$ and Agent $\times$ Congruency, $F(1,26)=3.04$, MSe $=$ $126.25, p>0.05$, partial $\eta^{2}=0.11$. However, we observed a significant effect of Gesture $\times$ Congruency, $F(2,52)=$ 18.55, MSe $=168.27, p<0.001$, partial $\eta^{2}=0.42$, indicating faster responses to complementary actions than to mirror actions for the communicative gesture, while transitive and intransitive gestures showed the typical congruency effect with faster responses for mirror actions than for complementary actions. Most importantly, we found a significant three-way interaction of Agent $\times$ Gesture $\times$ Congruency, $F(2,52)=4.14$, MSe $=141.28, p<0.05$, partial $\eta^{2}=$ 0.14 , indicating that the inverse congruency effect for the communicative gesture in the human agent condition amounting to $14 \mathrm{~ms}, t(26)=3.39, p<0.05$ was completely eliminated for the communicative gesture in the wooden condition, $t(26)=0.14, p=0.89$. This was not the case for the intransitive and transitive gesture conditions (see Fig. 3). For the intransitive gesture, we observed a congruency effect amounting to $12 \mathrm{~ms}, t(26)=4.41, p<0.001$ for the human agent condition and of $13 \mathrm{~ms}, t(26)=4.20$, $p<0.001$ for the wooden condition. For the transitive gesture, the congruency effect amounted to $12 \mathrm{~ms}$ for the human agent, $t(26)=3.72, p \leqslant 0.001$ and $9 \mathrm{~ms}$ for the wooden agent, $t(26)=2.27, p<0.05$.

\subsubsection{Error analysis}

We observed no main effect of Agent, $F(1,26)=1.35$, MSe $=5.71, p>0.05$, partial $\eta^{2}=0.05$, indicating that participants in the human and in the wooden condition did not differ in error rates. We found a main effect of Gesture, $F(2,52)=4.67, \mathrm{MSe}=5.15, p<0.05$, partial $\eta^{2}=0.15$, due to increased error rates for the intransitive gesture (1.9\%), as compared to the communicative (1.1\%) and the transitive (1.1\%) gestures. For errors, we found no significant effect of Congruency, $F(1,26)=3.08$, MSe $=3.92, p>0.05$, partial $\eta^{2}=0.11$. Further, we found no significant effects of Agent $\times$ Gesture $(F<1)$ and Agent $\times$ Congruency $(F<1)$. The interaction of Gesture $\times$ Congruency was significant, $F(2,52)=5.63, \quad \mathrm{MSe}=5.67, \quad p<0.05$, partial $\eta^{2}=0.18$, 
indicating the same trend as for RTs showing numerically fewer errors for the complementary action than for the mirror action for the communicative gesture, while the typical congruency effect was found for transitive and intransitive gestures, showing fewer errors for mirror actions than for complementary actions. Importantly, we found no significant three-way interaction of Agent $\times$ Gesture $\times$ Congruency $(F<1)$, indicating no evidence of a speed-accuracy trade-off (SAT) as an explanation for the observed RT effects.

\subsection{Discussion}

Using a variant of the classical motor priming paradigm, we were able to show clear compatibility effects for transitive and intransitive movements regardless of whether participants observed postures of a biological or a non-biological agent. In these two conditions, we found a compatibility effect of a similar size. Most importantly, however, we found a differential compatibility effect for observing a communicative gesture made by a human or non-human agent. A reversed compatibility effect was found when observing a human extending the right hand for a handshake. This reversal of the compatibility effect is not surprising given that we usually shake an extended right hand with our right hand, leading to spatial incompatibility of the relative position of the hand (see also Flach, Press, Badet, \& Heyes, 2010). For the non-human agent, however, this effect completely disappeared. This strongly indicates that the learned response to extend the right hand when observing a right hand is only activated when observing a human agent but not when observing this gesture made by a non-human agent.

While this finding is interesting in itself, extending the idea of a complementary action system to communicative behavior (Newman-Norlund, van Schie, van Zuijlen, \& Bekkering, 2007; Sebanz, Bekkering, \& Knoblich, 2006; van Schie, van Waterschoot, \& Bekkering, 2008), it may constrain the conclusions that can be drawn from the present experiment. The smaller motor priming effect for nonhuman actions might not be due to the communicative nature of the gesture, but rather to the specific complementary action used in Experiment 1 . Furthermore, direct hand-to-hand contact, which would be the usual response in such a situation, might lead to an aversive response when a non-human agent attempts to shake hands with you. This could alternatively explain the reduced motor priming effect in the specific non-human communicative action condition used in Experiment 1.

\section{Experiment 2}

We designed a second experiment in which we aimed to (a) replicate our findings of Experiment 1 and (b) test if the smaller motor priming effect for non-human actions is actually due to the communicative nature of the gesture or to its complementarity or aversive nature. Therefore, we created completely new stimulus material for the communicative gesture condition, which did not involve a complementary response or direct hand-to-hand contact. In

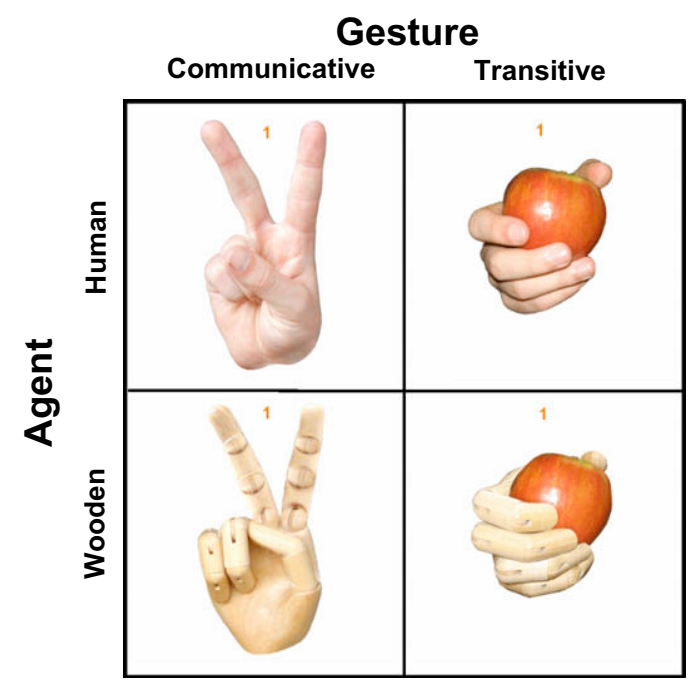

Fig. 4. The figures show the stimuli and design of two agents (human, wooden) and two gesture types (communicative and transitive) used in Experiment 2. All stimuli are shown from a third person perspective displaying a right hand in a mirror-congruent condition.

Experiment 2, we presented either a human or a wooden hand making a victory sign (see Fig. 4). We also used the transitive action stimuli from Experiment 1 as a control condition. In line with the findings from Experiment 1, we predicted smaller motor priming effects for non-human actions as compared to human actions for the communicative gesture, but similar positive motor priming effects for the transitive gesture condition for both agents.

\subsection{Method}

\subsubsection{Participants}

A new group of 32 undergraduate students (16 males, age range: $22-42$, mean age: 26.2 , SD: 4.5 ) participated in this experiment. All participants were right-handed, had normal or corrected-to-normal vision, and were naive with regard to the hypotheses of the experiment. Participants were paid $€ 7$ for participation.

\subsubsection{Apparatus and stimuli}

Apparatus and Stimuli were the same as used in Experiment 1, except that we omitted the intransitive gesture condition of Experiment 1. The communicative gesture stimuli were replaced by hand stimuli making a victory sign, photographed from a third person perspective (see Fig. 4). The same gesture was presented depicting two different agents (human and wooden). At a viewing distance of $80 \mathrm{~cm}$, the communicative hand on the screen subtended a visual angle $6.77^{\circ} \times 13.36^{\circ}$.

\subsubsection{Procedure and design}

Procedure and design were identical to Experiment 1. We used only communicative and transitive gestures in Experiment 2, meaning that participants performed 256 trials in total. 


\subsection{Results}

The same outlier procedure as in Experiment 1 was applied to the data set from Experiment 2, which resulted in the exclusion of $0.9 \%$ incorrect trials and $1.0 \%$ outliers. Congruency effects for all conditions are presented in Fig. 5. For an overview of mean RTs and mean percent errors of all conditions of Experiment 2, including main effects, see Table 2.

\subsection{1. $R T$ analysis}

We used a $2 \times 2 \times 2$-factorial design including the 2-level factor Agent (human, wooden), the 2-level factor Gesture (communicative, transitive), as well as the 2-level factor Congruency (congruent, incongruent), with all factors as within-subject variables (see Figs. 4 and 5). The same analysis was conducted for accuracy data.

We found no main effect of Agent, $F(1,31)=1.21$, MSe $=153.09, p>0.05$, partial $\eta^{2}=0.04$, indicating that RTs in the human and wooden conditions did not differ. We found no main effect of Gesture, $(F<1)$ indicating no differences in RTs between the transitive and the communicative gesture. Furthermore, we found a significant effect of Congruency, $F(1,31)=30.05$, MSe $=195.55, p<0.001$, partial $\eta^{2}=0.49$, due to faster RTs in congruent (403 ms) as compared to incongruent $(413 \mathrm{~ms})$ conditions. The interaction of Agent $\times$ Gesture approached significance, $F(1,31)=3.41, \quad \mathrm{MSe}=133.57, p=0.07$, partial $\eta^{2}=0.10$, showing a numerical trend of slower RTs in the wooden as compared to the human communicative condition. We observed no significant effect of Agent $\times$ Congruency, $(F<1)$, indicating that the basic congruency effects did not differ between the human and the wooden conditions. Furthermore, we found no significant effect of Gesture $\times$ Congruency, $F(1,31)=2.30, \mathrm{MSe}=163.28, p>0.05$, partial $\eta^{2}=0.07$, indicating no differences in the congruency effect between communicative and transitive gestures. Importantly, we found a significant three-way interaction of Agent $\times$ Gesture $\times$ Congruency, $F(1,31)=$ 4.35, $\mathrm{MSe}=105.97, p<0.05$, partial $\eta^{2}=0.12$, indicating
Table 2

Mean reaction times (RTs) in milliseconds (ms) and percent errors (upper part) are shown for Agent (human, wooden), Gesture (communicative, transitive) and Congruency (congruent, incongruent) conditions of Experiment 2. Corresponding $p$-values for within-subjects effects (lower part) are shown separately for RTs and percent errors.

\begin{tabular}{lll}
\hline Condition & RT (ms) & Error (\%) \\
\hline Human communicative congruent & 403 & 0.6 \\
Human communicative incongruent & 414 & 1.4 \\
Wooden communicative congruent & 411 & 0.8 \\
Wooden communicative incongruent & 415 & 1.0 \\
Human transitive congruent & 400 & 0.3 \\
Human transitive incongruent & 411 & 2.0 \\
Wooden transitive congruent & 398 & 1.0 \\
Wooden transitive incongruent & 411 & 0.5 \\
Within-subjects & $p(R T)$ & $p($ Error $)$ \\
\hline Agent $(\mathrm{A})$ & n.s. & n.s. \\
Gesture (G) & $n . s$. & $n . s$. \\
Congruency (C) & $<0.001^{\mathrm{b}}$ & $<0.05^{\mathrm{a}}$ \\
$\mathrm{A} \times \mathrm{G}$ & $n . s$. & $n . s$. \\
$\mathrm{A} \times \mathrm{C}$ & n.s. & $<0.001^{\mathrm{b}}$ \\
$\mathrm{G} \times \mathrm{C}$ & n.s. & n.s. \\
$\mathrm{A} \times \mathrm{G} \times \mathrm{C}$ & $<0.05^{\mathrm{a}}$ & n.s. \\
\hline
\end{tabular}

a Sign $(p<0.05)$

b Sign $(p<0.001)$, n.s. $(p>0.05)$.

that the significant congruency effect for the communicative gesture in the human agent condition amounting to $11 \mathrm{~ms}, t(31)=4.41, p<0.001$ was eliminated for the communicative gesture in the wooden condition, $t(31)=1.08$, $p=0.29$, while this was not the case for the transitive gesture conditions (see Fig. 5). We found a significant congruency effect of $10 \mathrm{~ms}, t(31)=3.17, p<0.05$ for transitive gestures in the human agent condition, as well as a $14 \mathrm{~ms}$ congruency effect for the transitive gesture in the wooden condition, $t(31)=4.35, p<0.001$.

\subsubsection{Error analysis}

For errors, only the factors Congruency, $F(1,31)=6.81$, MSe $=2.71, p<0.05$, partial $\eta^{2}=0.18$, and the interaction of Agent $\times$ Congruency $F(1,31)=15.99, \quad \mathrm{MSe}=1.87, \quad p<$

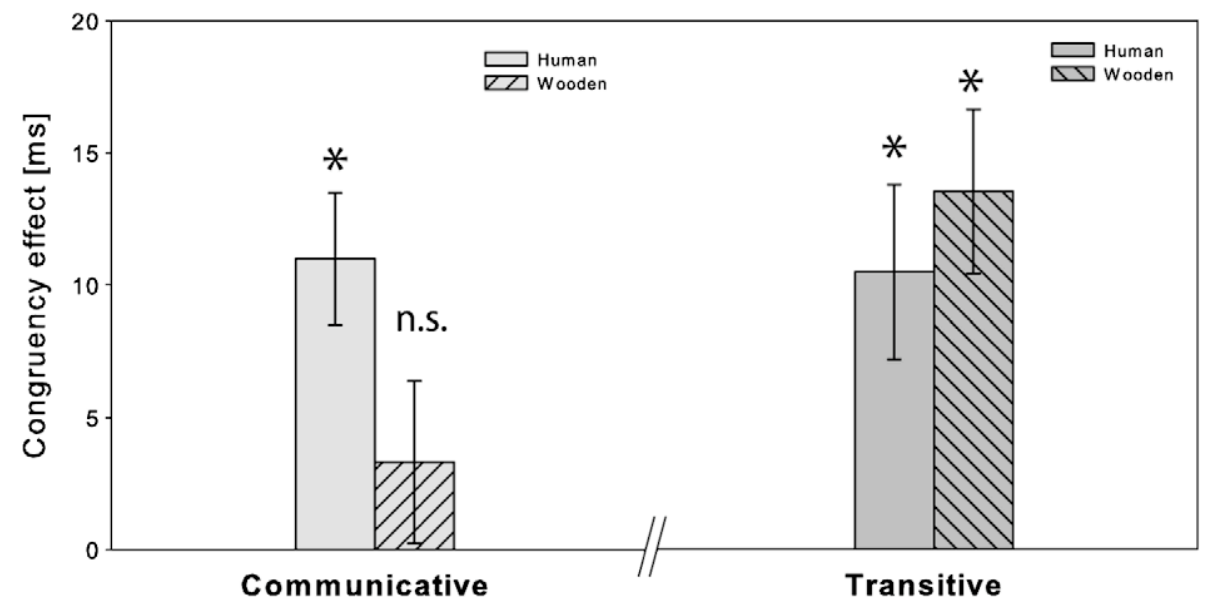

Fig. 5. Congruency effect (incongruent minus congruent) in milliseconds (ms) plotted with the SE of the congruency effect for Experiment 2 shown for communicative (left panel, light gray) and transitive gestures (right panel, dark gray) of the human agent (blank bars) and the wooden agent (striped bars). 
0.001 , partial $\eta^{2}=0.34$, reached significance, showing the typical congruency effect with fewer errors in congruent $(0.7 \%)$ than in incongruent (1.2\%) trials, which was mainly due to a congruency effect in the human condition. We found no evidence of a SAT as an explanation for the observed RT effects.

\subsection{Discussion}

Replicating the findings of Experiment 1, we show that human movement stimuli are more effective priming stimuli than non-human movement stimuli when involving a communicative gesture but not when involving a transitive gesture. We can also rule out the possibility that the complementarity or an aversive response to the non-human communicative action caused the differential effect for communicative and non-communicative actions in Experiment 1.

\section{General discussion}

Evidence from behavioral and neuroimaging research suggests that we simulate human agents and non-human agents differently in our motor system (Kilner et al., 2003; Liepelt \& Brass, 2010; Perani et al., 2001; Press, Gillmeister, \& Heyes, 2006; Press et al., 2005; Tai et al., 2004; Tsai \& Brass, 2007). The present study investigated whether motor simulation of non-human agents is modulated by the type of action observed. In accordance with previous literature, our results show that human movement stimuli produce stronger motor priming effects than non-human movement stimuli when the observed action is a communicative gesture. However, both human and non-human movement stimuli produced similar motor priming effects when the movement comprises a transitive or intransitive gesture. This shows that motor simulation is not only agent-specific, but also strongly interacts with the type of action the agent performs.

We believe that the present results extend previous findings by further qualifying the conditions under which humans simulate observed behavior. Previous research has demonstrated that whether we simulate observed behavior depends on the observed agent (e.g., Kilner et al., 2003; Tai et al., 2004). Furthermore, it has been demonstrated that simulation also seems to depend on whether the observed behavior belongs to the motor repertoire of the observer (Buccino et al., 2004). Our data suggest that whether we motorically simulate a behavior also depends on whether we consider this behavior as driven by a goal that is reasonable for the agent. We do not usually consider a communicative gesture to be part of a robot's action repertoire. On the other hand, we would consider a robot to be able to produce a simple hand-closing gesture or to grasp a glass. This dissociation strongly supports our previous claim that attribution of intentions to an observed agent is a crucial factor for motor simulation (Liepelt et al., 2008). Ochiai, Mushiake, and Tanji (2005) provided evidence in favour of goal simulation rather than simulation of the physical movement. They showed that monkey's premotor cortex responded not to the actual direction in which a limb must move, but to the visible direction of motion that is to be achieved. Furthermore, Bekkering and colleagues (Bekkering et al., 2000) were able to show that infants spontaneously imitate goals rather than physical movements. Our findings demonstrate that this goal bias already exists on an automatic motor simulation level.

The dissociation of instrumental and communicative actions is in line with a similar dissociation reported by Cole, Gallagher, and McNeill (2002) in a deafferented patient. This patient lost the sense of touch and motor control that both depend on proprioceptive feedback. While the patient was able to create language and communicative gesture patterns of normal complexity in timing and form, he had major problems controlling a movement, such as grasping a glass, without conscious visual attention.

\subsection{Similar motor priming effects when observing non- communicative gestures of human and non-human agents}

A surprising result of our study is the observation that transitive and intransitive actions produce the same motor priming effects regardless of whether one observes a human or a wooden hand. This seems to be at odds with previous behavioral results showing attenuation of motor priming effects when a non-biological agent was involved (Kilner et al., 2003; Press et al., 2005). There are several ways to explain this discrepancy. One way is a fundamental procedural difference between the experiments. While participants in previous studies observed congruent and incongruent movements while executing their response, the observed type of movement in the present study was always congruent. In our study, the motor priming effect is indirectly mediated via the activation of the left or right response hand. This would also explain why motor priming effects in previous experiments were substantially larger than in the present experiment. Further, previous studies seem to differ from the present study with respect to the dependent variable that was measured. Kilner et al. (2003), for example, measured the variance of a predefined movement while observing either congruent or incongruent movements. Furthermore, the non-human stimuli in our study were more similar to the human stimuli than they were in the study of Press and colleagues (2005) and Kilner and colleagues (2003). One could argue that it is more likely to attribute the intention to execute a transitive or intransitive action to a non-biological agent that is more similar to a human agent.

\subsection{Complementary action and motor priming}

In Experiment 1, we observed a reversed compatibility effect for the communicative gestures when participants observed a human hand, while no compatibility effect occurred when they observed the wooden hand. The reversal of the compatibility effect is an interesting finding in itself. It supports the idea that in some situations, complementary actions are the automatic response (Newman-Norlund, van Schie, van Zuijlen, \& Bekkering, 2007; van Schie et al., 2008). This strongly supports the idea that motor priming depends on learning (Catmur, Walsh, \& Heyes, 
2009; Catmur et al., 2008; Gillmeister, Catmur, Liepelt, Brass, \& Heyes, 2008). This finding is further in line with the findings of a previous study showing complementary priming effects induced by social handshake stimuli (Flach et al., 2010). When a specific behavior is contingent on a non-matching behavior, an incongruent association is formed. However, in the present context, this raises the possibility that the reduction in the motor priming effect for the non-human agent may not be related to the communicative nature of the gesture but rather to its complementarity. In the second Experiment, we were able to rule out this possibility. While Experiment 2 corroborates the lack of an agent-specific effect for the transitive gesture, it again shows a clear dissociation for the communicative gesture. This time the incongruent condition shows slower reaction times than the congruent condition when participants observed a human hand but not when observing a non-human hand.

\subsection{Effector specificity of motor priming}

One additional finding of the current study is that we observed effector-specific priming effects without confounding spatial compatibility. When observing a hand from a third person perspective, participants responded faster with the specular hand (e.g., model's right hand/observer's left hand) than with the anatomically matching hand (model's right hand/observer's right hand). The only exception was the handshake gesture, as discussed above. Since both left and right hands were presented centrally, we controlled for spatial compatibility. This finding is consistent with a brain imaging study by Koski and colleagues (Koski, Iacoboni, Dubeau, Woods, \& Mazziotta, 2003), who showed stronger mirror system activation when imitating in a specular way than in an anatomically matching way. Interestingly, however, Aziz-Zadeh and colleagues (2002) found stronger TMS evoked motor potentials in a passive observation task for the anatomically matching hand. In this study, stimuli were presented from the first person perspective (see also Liepelt et al., 2009). A study by Maeda, Kleiner-Fisman, and Pascual-Leone (2002) directly compared motor evoked potentials when observing a right hand from the first and the third person perspective. They found stronger motor evoked potentials in the right hand when observing a right hand movement from a first person perspective compared with a right hand movement from the third person perspective. Taken together, these findings suggest that when observing a hand from the third person perspective, the mirroring hand is activated but when observing a hand from the first person perspective, the anatomically matching hand is activated. The only study that is not consistent with this claim is a study by Press and colleagues (Press, Bird, Walsh, \& Heyes, 2008). They found the same motor priming effects when participants responded with their right hand regardless of whether they saw a right or a left hand from a first person perspective. However, we believe that the dependent measure was not sensitive enough in this study. Participants always had to respond with one hand and therefore small pre-activation of the hand was presumably not detectable.

\section{Conclusion}

In conclusion, the present study shows that it is the reasonableness of the apparent goal the observer attributes to the observed behavior that influences the observer's mental simulation process, such that those processes are evoked only when the observed behavior appears to be driven by a reasonable goal. On a more abstract level, our findings are in line with the assumption of top-down influences on motor simulation (Liepelt et al., 2008; Longo \& Bertenthal, 2009; Stanley et al., 2007).

\section{Acknowledgements}

This research was supported by a European funded project aimed at understanding the evolutionary, developmental and intentional control of imitation (EDICI12929). The authors wish to thank Margret Schött for help in data acquisition and Michael Masson for helpful comments on a previous version of the manuscript.

\section{References}

Aziz-Zadeh, L., Maeda, F., Zaidel, E., Mazziotta, J., \& Iacoboni, M. (2002). Lateralization in motor facilitation during action observation: A TMS study. Experimental Brain Research, 144, 127-131.

Bekkering, H., Wohlschlager, A., \& Gattis, M. (2000). Imitation of gestures in children is goal-directed. Quarterly Journal of Experimental Psychology A, 53, 153-164.

Beringer, J. (2000). Experimental Runtime System. BeriSoft Cooperation, Frankfurt am Main (1987-2000).

Bertenthal, B. I., Longo, M. R., \& Kosobud, A. (2006). Imitative response tendencies following observation of intransitive actions. Journal of Experimental Psychology: Human Perception and Performance, 32, 210-225.

Blakemore, S. J., \& Frith, C. (2005). The role of motor contagion in the prediction of action. Neuropsychologia, 43, 260-267.

Brass, M., Bekkering, H., Wohlschläger, A., \& Prinz, W. (2000). Compatibility between observed and executed finger movements: Comparing symbolic, spatial, and imitative cues. Brain Cognition, 44, 124-143.

Brass, M., \& Heyes, C. M. (2005). Imitation: Is cognitive neuroscience solving the correspondence problem? Trends in Cognitive Science, 9, 489-495.

Buccino, G., Binkofski, F., Fink, G. R., Fadiga, L., Fogassi, L., Gallese, et al. (2001). Action observation activates premotor and parietal areas in a somatotopic manner: An fMRI study. European Journal of Neuroscience, $13,400-404$.

Buccino, G., Lui, F., Canessa, N., Patteri, I., Lagravinese, G., Benuzzi, F., et al. (2004). Neural circuits involved in the recognition of actions performed by nonconspecifics: An fMRI study. Journal of Cognitive Neuroscience, 16, 114-126.

Candidi, M., Urgesi, C., Ionta, S., \& Aglioti, S. M. (2008). Virtual lesion of ventral premotor cortex impairs visual perception of biomechanically possible but not impossible actions. Social Neuroscience, 3, 388-400.

Catmur, C., Gillmeister, H., Bird, G., Liepelt, R., Brass, M., \& Heyes, C. M. (2008). Through the looking glass: Counter-mirror activation following incompatible sensorimotor learning. European Journal of Neuroscience, 28, 1208-1215.

Catmur, C., Walsh, V., \& Heyes, C. (2009). Associative sequence learning: The role of experience in the development of imitation and the mirror system. Philosophical Transactions of the Royal Society Series B Biological Sciences, 364, 2369-2380.

Cole, J., Gallagher, S., \& McNeill, D. (2002). Gesture following deafferentation: A phenomenologically informed experimental study. Phenomenology and the Cognitive Sciences, 1, 49-67.

Flach, R., Press, C., Badet, A., \& Heyes, C. M. (2010). Shaking hands: Priming by social effects. British Journal of Psychology. doi:10.1348/ $000712609 \times 484595$.

Fogassi, L., Ferrari, P. F., Gisierich, B., Rozzi, S., Chersi, F., \& Rizzolatti, G. (2005). Parietal lobe: From action organisation to intention understanding. Science, 308, 662-667. 
Gallese, V., Fadiga, L., Fogassi, L., \& Rizzolatti, G. (1996). Action recognition in the premotor cortex. Brain, 119, 593-609.

Gazzola, V., Aziz-Zadeh, L., \& Keysers, C. (2006). Empathy and the somatotopic auditory mirror system in humans. Current Biology, 16, 1824-1829.

Gazzola, V., Rizzolatti, G., Wicker, B., \& Keysers, C. (2007). The anthropomorphic brain: The mirror neuron system responds to human and robotic actions. NeuroImage, 35, 1674-1684.

Gillmeister, H., Catmur, C., Liepelt, R., Brass, M., \& Heyes, C. M. (2008). Experience-based priming of body parts: A study of action imitation. Brain Research, 1217, 157-170.

Grezes, J., Armony, J. L., Rowe, J., \& Passingham, R. E. (2003). Activations related to "Mirror" and "Canonical" neurones in the human brain: A fMRI study. NeuroImage, 18, 928-937.

Iacoboni, M., Woods, R. P., Brass, M., Bekkering, H., Mazziotta, J. C., \& Rizzolatti, G. (1999). Cortical mechanisms of human imitation. Science, 286, 2526-2528.

Jansson, E., Wilson, A. D., Williams, J. H., \& Mon-Williams, M. (2007). Methodological problems undermine tests of the ideo-motor conjecture. Experimental Brain Research, 182, 549-558.

Kilner, J., Paulignan, Y., \& Blakemore, S. (2003). An interference effect of observed biological movement on action. Current Biology, 13, 522-525.

Koski, L., Iacoboni, M., Dubeau, M. C., Woods, R. P., \& Mazziotta, J. C. (2003). Modulation of cortical activity during different imitative behaviors. Journal of Neurophysiology, 89, 460-471.

Liepelt, R., \& Brass, M. (2010). Top-down modulation of motor priming by belief about animacy. Experimental Psychology. doi:10.1027/16183169/a000028.

Liepelt, R., Ullsperger, M., Obst, K., Spengler, S., von Cramon, D. Y., \& Brass, M. (2009). Contextual movement constraints of others modulate motor preparation in the observer. Neuropsychologia, 47, 268-275.

Liepelt, R., von Cramon, D. Y., \& Brass, M. (2008). What is matched in direct matching? Intention attribution modulates motor priming. Journal of Experimental Psychology: Human Perception and Performance, 34, 578-591.

Longo, M. R., \& Bertenthal, B. I. (2009). Attention modulates the specificity of automatic imitation to human actors. Experimental Brain Research, 192, 739-744.

Longo, M. R., Kosobud, A., \& Bertenthal, B. I. (2008). Automatic imitation of biomechanically impossible movements: Effects of priming movements vs. goals. Journal of Experimental Psychology: Human Perception and Performance, 34, 489-501.

Maeda, F., Kleiner-Fisman, G., \& Pascual-Leone, A. (2002). Motor facilitation while observing hand actions: Specificity of the effect and role of observer's orientation. Journal of Neurophysiology, 87, 1329-1335.

Newman-Norlund, R., van Schie, H. T., van Zuijlen, A., \& Bekkering, H. (2007). The mirror neuron system is more active during complementary compared with imitative action. Nature Neuroscience, 10, 817-818.

Ochiai, T., Mushiake, H., \& Tanji, J. (2005). Involvement of the ventral premotor cortex in controlling image motion of the hand during performance of a target-capturing task. Cerebral Cortex, 15, 929-937.

Perani, D., Fazio, F., Borghese, N. A., Tettamanti, M., Ferrari, S., Decety, J., et al. (2001). Different brain correlates for watching real and virtual hand actions. NeuroImage, 14, 749-758.

Press, C., Bird, G., Flach, R., \& Heyes, C. (2005). Robotic movement elicits automatic imitation. Cognitive Brain Research, 25, 632-640.

Press, C., Bird, G., Walsh, E., \& Heyes, C. M. (2008). Automatic imitation of intransitive actions. Brain \& Cognition, 67, 44-50.

Press, C., Gillmeister, H., \& Heyes, C. (2006). Bottom-up, not top-down, modulation of imitation by human and robotic models. European Journal of Neuroscience, 24, 2415-2419.

Sebanz, N., Bekkering, H., \& Knoblich, G. (2006). Joint actions: Bodies and minds moving together. Trends in Cognitive Sciences, 10, 70-76.

Stanley, J., Gowen, E., \& Miall, R. C. (2007). Effects of agency on movement interference during observation of a moving dot stimulus. Journal of Experimental Psychology: Human Perception and Performance, 33, 915-926.

Stürmer, B., Ascherschleben, G., \& Prinz, W. (2000). Correspondence effects with manual gestures and postures: A study of imitation. Journal of Experimental Psychology: Human Perception and Performance, 26, 1746-1759.

Tai, Y. F., Scherfler, C., Brooks, D. J., Sawamoto, N., \& Castiello, U. (2004). The human premotor cortex is 'mirror' only for biological actions. Current Biology, 14, 117-120.

Tsai, C. C., \& Brass, M. (2007). Does the human motor system simulate Pinocchio's actions? Co-acting with a human hand versus a wooden hand in a dyadic interaction. Psychological Science, 18, $1058 \mathrm{U} 1062$.

Tucker, M., \& Ellis, R. (1998). On the relations between seen objects and components of potential actions. Journal of Experimental Psychology: Human Perception and Performance, 24, 830-846.

van Schie, H. T., van Waterschoot, B. M., \& Bekkering, H. (2008). Understanding action beyond imitation: Reversed compatibility effects of action observation in imitation and joint action. Journal of Experimental Psychology: Human Perception and Performance, 34, 1493-1500. 